\title{
Successful Treatment of a Cervical Ectopic Pregnancy with Single-Dose Methotrexate Therapy
}

\author{
Hannah Dearworth, MD ${ }^{1}$, Jesse Cottrell, MD ${ }^{1}$
}

ABSTRACT

Ectopic pregnancies implanted in the cervix account for less than one percent of all extra-uterine pregnancies. Due to the rare incidence of cervical ectopic pregnancies, there are no established guidelines for medical versus surgical management. We report a case of a cervical ectopic pregnancy with a fetal heartbeat successfully treated with single-dose methotrexate therapy.

\section{KEYWORDS}

Cervical Ectopic Pregnancy, Methotrexate, Pregnancy
Author affiliations are listed at the end of this article.

Correspondence to: Hannah Dearworth, MD Marshall University Joan C. Edward School of Medicine johnsonha@marshall.edu

\section{INTRODUCTION}

An ectopic pregnancy is a pregnancy that is implanted outside of the uterus, most commonly within the fallopian tube, but can occur in other rare sites such as the cervical canal. Cervical ectopic pregnancies occur in 1 in 9000 pregnancies. ${ }^{1,2}$ An ectopic pregnancy implanted in the cervical canal is considered a non-viable gestation with high risk for maternal morbidity and mortality. Prior reports describe multi-dose methotrexate therapy for an embryo with no fetal pole, and direct potassium chloride $(\mathrm{KCL})$ injection into the gestational sac if a heartbeat is present. ${ }^{3}$ We report a case of a cervical ectopic pregnancy with a fetal heartbeat successfully treated with single-dose methotrexate therapy.

\section{CASE DESCRIPTION}

\section{A 22-year-old gravida 2 para 0 at 6 weeks and 5} days gestational age by the last menstrual period presented to the emergency room with heavy vaginal bleeding. A previous dating and viability transvaginal ultrasound at her OB/GYN's office was suspicious for a cervical ectopic pregnancy. She reported no previous medical conditions. The patient's obstetric history was significant for a previous dilation and curettage at 7 weeks and 3 days gestation due to a spontaneous abortion. She denied any sexually transmitted infections.

On physical exam, the patient's abdomen was soft, non-tender and non-distended. Initial vital signs were temperature 97.6 F, pulse 77, blood pressure 109/68. Sterile speculum exam was performed, and clotted blood was observed protruding from the external cervical os. Transvaginal ultrasound was performed and demonstrated an intracervical pregnancy with a crown-rump length of $6.8 \mathrm{~mm}$, a gestational sac measuring $9.9 \mathrm{~mm}$, a yolk sac measuring $3.0 \mathrm{~mm}$, and a fetal heart rate of 113 beats per minute (Figure 1 and Figure 2). A maternal-fetal medicine specialist was consulted, and the diagnosis of a cervical ectopic pregnancy was confirmed. The patient's left and right ovaries were visualized and appeared normal with a suspected right corpus luteum cyst. The initial beta-human chorionic gonadotropin (HCG) quantitative measurement was $5688 \mathrm{mlU} / \mathrm{mL}$. The initial hemoglobin/hematocrit ( $\mathrm{Hgb} / \mathrm{Hct}$ ) level was $14.0 / 42.1$ and the patient was typed and crossmatched. 


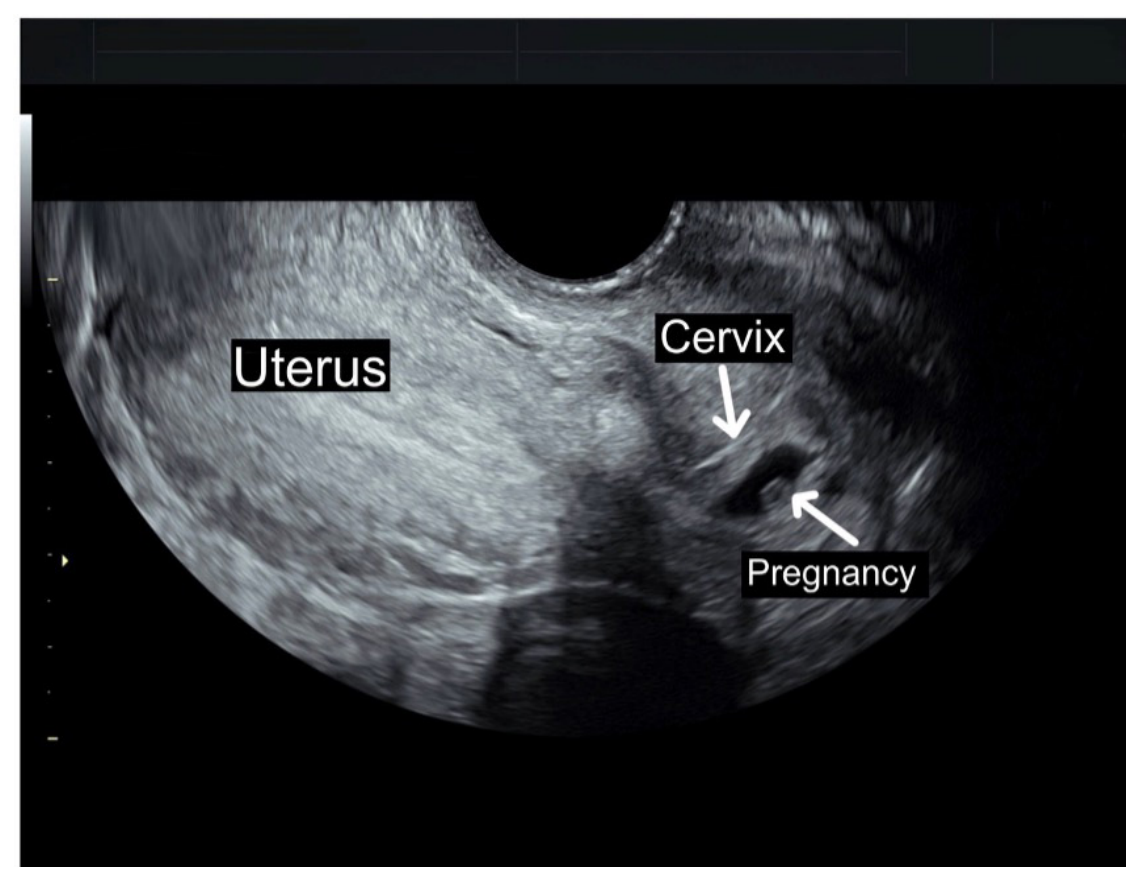

FIGURE 1: Pregnancy Implanted in the Cervical Canal

After counseling the patient on the ultrasound and laboratory findings, routes of administration were discussed. These included singledose methotrexate, multiple-dose methotrexate or $\mathrm{KCL}$ injection for a cervical ectopic pregnancy. She elected to proceed with single-dose methotrexate therapy. Methotrexate $50 \mathrm{mg} / \mathrm{m} 2$ was given to the patient, for a total dose of $84.5 \mathrm{mg}$.

One day following methotrexate administration (post-injection day 1 ), cardiac activity was again demonstrated on ultrasound, and repeat betaHCG quantitative measurement was $5587 \mathrm{mlU} /$ $\mathrm{mL}$. Following the ultrasound, the patient began experiencing nausea and then heavy vaginal bleeding requiring the use of three pads over six hours. She never became hemodynamically unstable, and repeat measurement of $\mathrm{Hgb} / \mathrm{Hct} 12.2 / 36.6$ was reassuring. A transvaginal ultrasound performed the following morning (post-injection day 2) showed no evidence of intracervical pregnancy. Her beta-HCG quantitative measurement decreased to $1856 \mathrm{mIU} /$ $\mathrm{mL}$. The patient then signed out against medical advice.

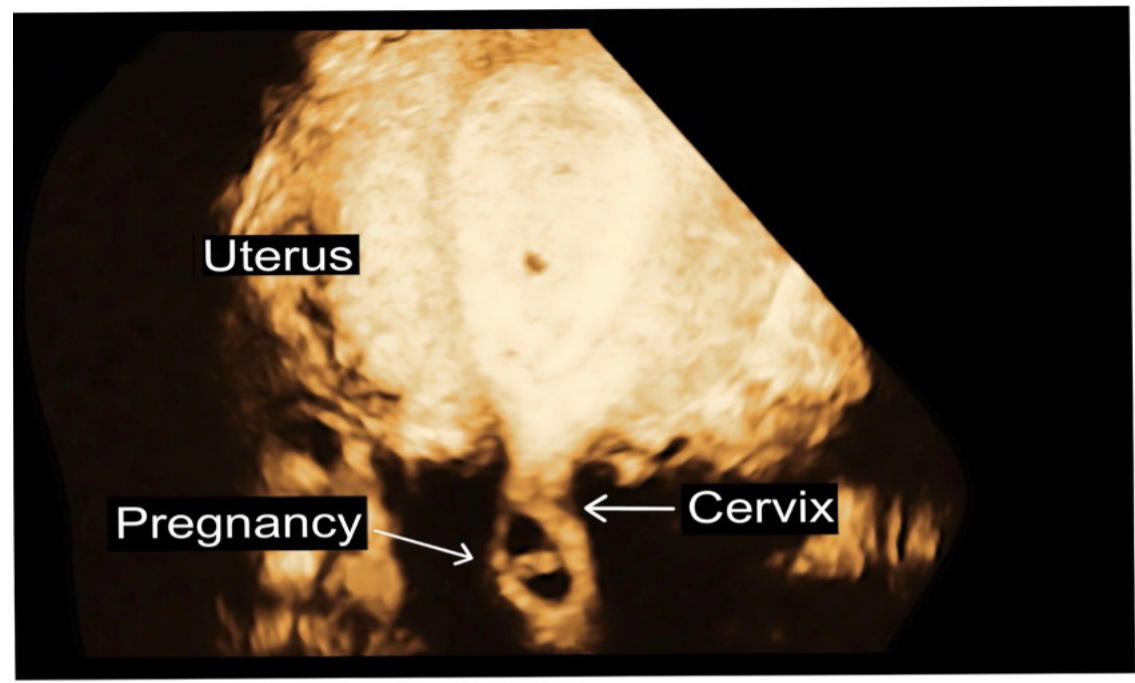

FIGURE 2: 3D Rendering of Pregnancy Implanted in the Cervical Canal

\section{DISCUSSION}

Cervical ectopic pregnancies account for less than 1 percent of all ectopic pregnancies. ${ }^{4,5}$ Early diagnosis and treatment are critical in cervical pregnancies due to the high risk of maternal morbidity and mortality, primarily due to severe hemorrhage. ${ }^{6}$

The most common presenting symptom of a cervical ectopic pregnancy is vaginal bleeding, which is often 
painless and profuse. The differential diagnosis for painless vaginal bleeding in early pregnancy includes ectopic pregnancy, miscarriage, implantation bleeding, and gestational trophoblastic disease. ${ }^{7}$

Management is primarily dictated by the patient's hemodynamic status. In a stable patient, the mainstay of diagnosis is transvaginal ultrasound and physical exam coupled with serial serum beta HCG levels following treatment. Due to the rare incidence of cervical ectopic pregnancies, there are no established guidelines for medical versus surgical management. Existing literature from case reports and case series suggests initial treatment with multi-dose methotrexate therapy if there is no fetal heartbeat, and direct intraamniotic potassium chloride (KCL) injection if a fetal heartbeat is present. ${ }^{8}$ While methotrexate has a higher chance of failure, intra-amniotic KCL injection is associated with maternal hemorrhage when the gestational sac collapses. ${ }^{9}$ Methotrexate interferes with DNA synthesis, repair, and cellular replication by inhibiting DHFR, which depletes the cellular pools. Some absolute contraindications to methotrexate therapy are active pulmonary disease, alcoholism, breastfeeding, hematologic dysfunction, immunodeficiency syndrome, peptic ulcer disease and renal disease. ${ }^{10}$

In fallopian tube ectopic pregnancies, two-dose methotrexate is recommended if the beta-HCG quantitative is above $3,000 \mathrm{mlU} / \mathrm{mL}$ or if the mass measures greater than two $\mathrm{cm}$. Multi-dose methotrexate is another option for cervical ectopic pregnancies and requires $1 \mathrm{mg} / \mathrm{kg}$ per day on days 1 , 3,5 , and 7 combined with IM leucovorin $0.1 \mathrm{mg} / \mathrm{kg}$ on days $2,4,6$, and 8 . A single dose approach is less expensive, requires less intensive monitoring and has fewer adverse effects. ${ }^{11}$ In this current case, our patient elected for a more conservative management with single-dose methotrexate therapy. In light of the patient's stable hemodynamic status and ability to provide inpatient monitoring during and after treatment with methotrexate, this approach with methotrexate therapy alone was considered a reasonable option in this case. An early gestational age of 6 weeks and 5 days could have contributed to the success of single-dose methotrexate therapy in this case. A limitation in this case was that the beta-HCG levels could not be followed to zero due to the patient leaving against medical advice. Repeat imaging demonstrated resolution of the cervical ectopic pregnancy, and repeat beta-HCG levels significantly declined. Serial HCG levels should be obtained until a nonpregnancy level is achieved, which may take up to eight weeks. Failure of the HCG level to decrease by at least $15 \%$ from day four to day seven after methotrexate administration is associated with treatment failure and additional methotrexate or surgical intervention may be considered..$^{10}$

Cervical ectopic pregnancies are uncommon. When counseling patients regarding their options for the treatment of cervical ectopic pregnancies, a shared decision-making model should be employed when determining how to treat these non-viable pregnancies. A conservative approach with singledose methotrexate therapy was successful in this case even though a fetal heartbeat was present on ultrasound.

\section{AUTHOR AFFILIATIONS}

1. Marshall University Joan C. Edwards School of Medicine, Huntington, West Virginia

\section{REFERENCES}

1. Ushakov FB, Elchalal U, Aceman PJ, Schenker JG. Cervical pregnancy: past and future. Obstet Gynecol Surv. 1997;52(1):45-59.

2. Singh S. Diagnosis and management of cervical ectopic pregnancy. J Hum Reprod Sci. 2013;6:273-276.

3. Jeng $\mathrm{CJ}$ et al. Transvaginal ultrasound-guided treatment of cervical pregnancy. Obstet Gynecol. 2007;109(5): 1076-1082.

4. Dolinko AV, Vrees RA, Frishman GN. Non-tubal ectopic pregnancies: overview and treatment via local injection. Journal of Minimally Invasive Gynecology. 2018;25(2):287-296.

5. Bouyer J et al. Sites of ectopic pregnancy: a 10 year population-based study of 1800 cases. Hum Reprod. 2002;17(12): 3224-3230.

6. Munoz JL, Kalan A, Singh K. Second trimester cervical ectopic pregnancy and hemorrhage: a case report and review of the literature. Case Rep Obstet Gynecol. 2018:3860274. 
7. Tulandi T. Cervical pregnancy. UpToDate. 2019.

8. Celik C, Bala A, Acar A, Gezgine K, Akyurek C. Methotrexate for cervical pregnancy. A case report. J Reprod Med. 2003;48:130-32.

9. Schufreider $A$, et al. Successful termination of a cesarean scar ectopic pregnancy with systemic methotrexate and local potassium chloride injection. J Reproductive Endocrinology \& Infertility. 2016;1:8.

10. Tubal ectopic pregnancy. ACOG Practice Bulletin No. 193. American College of Obstetricians and Gynecologists. Obstet Gynecol 2018;131:e91-103

11. Tunlandi T. Ectopic pregnancy: methotrexate therapy. UpToDate. 2020. 